Trauma Berufskrankh 2014 · 16[Suppl 1]:26-30

DOI 10.1007/s10039-013-1959-0

Online publiziert: 27. Juni 2013

๑) Springer-Verlag Berlin Heidelberg 2013
F. Schröter

Interdisziplinäre Begutachtungen, IMB Kassel

\section{Kommunikation zwischen Arzt und Patient} Aspekte bei der Begutachtung

\section{Kommunikationsprobleme}

\section{Zwischen Arzt und Patient}

Sie sind zweifellos der Entwicklung in der modernen Medizin geschuldet und dürften sich erst in der Nachkriegsära nach und nach entwickelt haben. Die Gründe hierfür sind vielfältiger Natur. Mitte des vorigen Jahrhunderts wurde - von Ausnahmen abgesehen - nur der wirklich kranke Patient beim Hausarzt vorstellig, der in der Regel auch die sozioökonomischen Hintergründe seiner $\mathrm{Pa}$ tienten kannte und eine sprechende $\mathrm{Me}$ dizin pflegte [1], den Patienten über ein gesundheitsgerechtes Verhalten instruierte und als angesehene Autorität auch durchsetzen konnte. Nur relativ wenige Patienten bedurften der Weiterleitung zum Facharzt, der mit wenigen Patienten ebenfalls begleitend zu dem eingeschlagenen fachmedizinisch begründeten therapeutischen Weg noch Zeit hatte für eine begleitende sprechende Medizin, sich also auch der Sorgen und Nöte seines Patienten annehmen konnte.

Durch den zwischenzeitlich gewaltigen medizinisch-technischen Wissenszuwachs und damit einer seinerzeit ungeahnten Eröffnung neuer und nachhaltig wirksamer Behandlungsmethoden einerseits, aber auch die zwischenzeitlich entstandenen Neigungen in der Bevölkerung, schon mit geringen unklaren Beschwerden den Arzt - insbesondere den Facharzt direkt - aufzusuchen, wurde die sprechende Medizin schlichtweg zu einem Zeitproblem. Kommunikation zwi- schen Arzt und Patient wurde gewissermaßen zu einem - nicht genügend honorarfähigen - Luxus. Der Arzt operiert zudem mit seinem Fachwissen weit über der durchschnittlichen Auffassungsgabe der Patienten, was einen informellen Dialog zwischen Arzt und Patient - gewissermaßen auf Augenhöhe - erschwert. Der Arzt versteckt sich gleichsam hinter seiner Fachautorität, anstatt den Patienten mit dieser zu begleiten und im Umgang mit seiner Erkrankung zu führen, wie dies vor einem guten halben Jahrhundert noch üblich war.

\section{Zwischen Gutachter und Proband}

Diese Kommunikationsprobleme sind leider nicht selten auch im Bereich der Begutachtungsmedizin zu finden und werden von manchen Probanden auch thematisiert, teils mit fast unglaublich anmutenden Erlebnissen, wie die folgenden Fallbeispiele, die keiner Kommentierung bedürfen, zeigen.

\section{Fall 1}

Eine 51-jährige Lehrerin wurde nach Verheilen eines Sprunggelenkbruchs - erlitten im Sportunterricht - zur Erstellung eines Gutachtens in eine orthopädische Praxis geschickt. Dort wurde sie freundlich empfangen und von einer sehr jungen Mitarbeiterin - die sich als Auszubildende vorstellte - mit einem Laptop unter dem Arm in einen kleinen Raum geführt, wo ihre Personalien direkt in den Computer eingetippt wurden. Anschließend wurde - zur Verblüffung der Lehrerin - von der Auszubildenden auch die Anamnese erhoben bis hin zum verbliebenen Beschwerdebild durch den Sprunggelenkbruch. Sämtliche Daten wurden direkt in den Computer eingegeben und die Lehrerin nach Abschluss des Gesprächs gebeten, im Wartezimmer Platz zu nehmen.

Nach kurzer Wartezeit wurde die Frau aufgerufen, vom Arzt freundlich begrüßt und umgehend gebeten, sich zu entkleiden und auf der Untersuchungsliege Platz zu nehmen.

Der Arzt ermittelte die Messdaten (Umfangsmaße und Bewegungsgrade), die er ebenfalls direkt in seinen Computer eingab, und verabschiedete sich mit der Instruktion, im Wartezimmer auf die noch notwendige Röntgenbilddiagnostik zu warten. Ein ärztliches Gespräch zum Verletzungsbild und den Folgen fand nicht statt. Versuche der Lehrerin, sich entsprechend gegenüber dem Arzt zu artikulieren, wurden mit der Information abgeblockt, dass dies alles schon im Computer nachzulesen sei.

Der Arztkontakt soll nach Angaben der Lehrerin keine 5 min gedauert haben. Sie beschwerte sich später bei der Berufsgenossenschaft über diese Art der Durchführung des Gutachtens und verlangte ein weiteres Gutachten, da sie bei einer solchen Abwicklung kein Vertrauen in die Richtigkeit der gutachtlichen Expertise habe.

\section{Fall 2}

Ein Lagerarbeiter fing eine aus einem Regal heraus fallende Last (Gewicht ungefähr $30 \mathrm{~kg}$ ) auf und klagte seitdem über 
Schulterschmerzen. Am Folgetag suchte er einen D-Arzt (Durchgangsarzt) auf.

Am Empfang fragte die Sprechstundenhilfe, wie der Schulterschmerz entstanden sei, und begab sich in einen anderen Raum, um mit dem Arzt zu sprechen. Sie kehrte mit der Mitteilung zurück, dass sich laut Auffassung des D-Arztes kein Arbeitsunfall ereignet hätte und der Mann nur nach Zahlung der Praxisgebühr von 10 EUR beim Arzt vorstellig werden könne. Ansonsten müsse er eine Überweisung vom Hausarzt mitbringen, der jedoch über $100 \mathrm{~km}$ entfernt ansässig war. Der Proband verließ empört die DArzt-Praxis und stellte sich nach Rückkehr an seinen Wohnort am nächsten Tag beim Hausarzt vor, der ihm eröffnete, dass er hierfür nicht zuständig sei, sondern der Patient zum D-Arzt müsse.

Ein Telefonat mit einem D-Arzt in der Nähe führte zur Empfehlung, zunächst eine MRT-Untersuchung (MRT: Magnetresonanztomographie) in die Wege zu leiten und den Patienten erst anschließend zur D-ärztlichen Untersuchung vorzustellen. Für die Wartezeit solle der Hausarzt ein Analgetikum verordnen. Die MRT-Untersuchung wurde 11 Tage später durchgeführt, weitere 6 Tage später wurde der D-Arzt-Termin wahrgenommen. Der D-Arzt empfing den Patienten mit den Worten, dass er schon Bescheid wisse, was passiert sei, und ließ sich die MRTAufnahmen geben. Als der Patient Anstalten machte, den Oberkörper zu entkleiden, äußerte sich der D-Arzt dahingehend, dass dies gar nicht notwendig sei. Er könne an den Bildern erkennen, dass er operieren müsse, der Patient solle sich am Empfang einen Termin für eine ambulante Arthroskopie geben lassen.

\section{Kommunikation im ärztlichen Alltag}

Gesellschaftliche Grundvorstellungen bestimmen auch - und nachhaltig - den Umgang zwischen Arzt und Patient miteinander. Hierzulande gehen sie auf christliche und humanistische Werte zurück, unterliegen jedoch auch einem Wandel durch den Zeitgeist. Die Gesellschaft geht heute z. B. ganz anders mit Kindern um, also noch vor 100 Jahren. Wurden sie seinerzeit noch erzogen, bemüht man sich heute um die Entwicklung zu einer eigenverantwortlich handelnden Persönlichkeit.

Vor 100 Jahren war ein paternalistisches Verhältnis zwischen väterlich-fürsorglich agierendem Arzt und seinem mit medizinischen Kenntnissen nicht vertrauten Patienten eine Selbstverständlichkeit. Die Ansage des Arztes wurde widerspruchslos befolgt!

Dieses paternalistische Selbstverständnis der Ärzte ist zumindest latent immer noch erkennbar, obwohl - nicht zuletzt auch durch die Gesetzgebung und die Rechtssprechung - zu Recht ein Partnerschaftsmodell im Umgang zwischen Arzt und Patient gefordert wird: Der Arzt informiert seinen Patienten über seine Erkrankung und die damit verknüpften prognostischen Aspekte, über die alternativen Möglichkeiten der Behandlung, er hilft dem Patienten beratend, zu einer eigenen Bewertung und Entscheidung zu gelangen, was aber nur dann funktionieren kann, wenn die medizinischen Aspekte so kommuniziert werden, dass sie vom Patienten auch verstanden werden können. Im Idealfall wird der derart aufgeklärte Patient dem Rat seines Arztes folgen, der andererseits eine gegenteilige $\mathrm{Pa}-$ tientenentscheidung zu respektieren hat.

Auch bei dem sog. Konsumentenmodell der Arzt-Patient-Beziehung bedarf es zunächst einer dann eher einseitigen Kommunikation mit Vorstellung unterschiedlicher Behandlungsmöglichkeiten durch den Arzt, der sich mehr als technischer Experte versteht, nicht unbedingt berät, sondern die Entscheidungen allein dem sog. Konsumenten, also dem Patienten, überlässt. Dieser Art der Arzt-Patienten-Beziehung wohnt jedoch die latente Gefahr inne, dass nicht die sinnvollste, sondern die teuerste Behandlungsmethode vermittelt wird, wie dies z. B. im zahnärztlichen Bereich (Zahnersatz) zumindest bei Privatpatienten inzwischen gang und gäbe ist. In diese Kategorie wird man auch das sog. IGeLn (IGeL: individuelle Gesundheitsleistungen) der Ärzte einzuordnen haben.

\section{Kommunikation bei der Begutachtung}

\section{Hintergrund}

Der ärztliche Sachverständige bewegt sich in einem gänzlich anderen Rollenverständnis, als dies im kurativen Bereich der Fall ist. Von Ausnahmen abgesehen muss er nicht neu diagnostizieren und Behandlungsstrategien vermitteln, sondern bereits ermittelte Befunde und Diagnosen bezüglich ihrer Ursächlichkeit hinterfragen und die gesundheitlichen Verhältnisse des ihm anvertrauten Probanden - man spricht im gutachtlichen Bereich nicht von einem Patienten - gewichten, um daraus z. B. eine MdE- (Minderung der Erwerbsfähigkeit) oder Invaliditätsbemessung (gesetzliche und private Unfallversicherung) oder einen Grad der Behinderung (Schwerbehindertenrecht) abzuleiten.

Es besteht kein stillschweigender Dienstleistungsvertrag zwischen Arzt und Patient, sondern nur ein Werkvertrag zwischen Gutachter und Auftraggeber, der den Sachverständigen verpflichtet, die gutachtliche Beurteilung handwerklich mit sorgfältigem Abarbeiten in der richtigen Reihenfolge der Prüfkriterien einwandfrei zu erstellen.

Trotz strikter Neutralität gegenüber allen Beteiligten im Prüfungsverfahren steht der gutachtlich tätige Arzt dennoch ethisch in der hippokratisch geprägten Pflicht, den ihm anvertrauten Menschen vor Schaden und willkürlichem Unrecht zu bewahren.

Dieses Grundanliegen eines jeden ärztlichen Sachverständigen sollte auch dem ihm anvertrauten Menschen vermittelt werden, wozu es einer recht umfangreichen Kommunikation mit dem Probanden bedarf. So verlangt die Sozialgesetzgebung vom gutachtlich tätigen Arzt, den Patienten über Sinn und Zweck des gutachtlichen Prozederes aufzuklären, ihn über seine Rechte - auch der möglichen Verweigerung z. B. von Röntgenuntersuchungen - zu informieren, aber auch über seine Pflichten der Mitwirkung.

Erfahrungsgemäß ergibt sich hieraus zu Beginn des Arzt-Patienten-Gespräches ein lebhafter Dialog mit zusätzlichen Fragen des Probanden, was - wenn 
vom Arzt respektvoll durchgeführt - einer ganz wesentlichen vertrauensbildenden Maßnahme in der Begutachtungssituation gleichkommt. Gelingt es dem ärztlichen Sachverständigen seinem ihm anvertrauten Probanden zu vermitteln, dass er nicht der Sachverständige der auftraggebenden Versicherung ist, sich dieser Aspekt lediglich auf die Kostenübernahme beschränkt, sondern alle Argumente des Probanden empathisch aufnehmen und zu prüfen hat, kann sich auch in einer so kurzen Begegnung zwischen dem Probanden und dem ärztlichen Sachverständigen ein Vertrauensverhältnis entwickeln, welches schlussendlich der Akzeptanz der gutachtlichen Beurteilung dient, insbesondere dann, wenn diese nicht den Vorstellungen des Probanden entspricht.

\section{Begutachtung}

\section{Einladung}

Die angeführten vertrauensbildenden Maßnahmen beginnen bereits mit dem ersten Kontakt zum Probanden, nämlich mit einer freundlich formulierten Einladung zur gutachtlichen Untersuchung inklusive Informationen zur Anreise und zur eventuellen Kontaktaufnahme mit einem kompetenten Ansprechpartner, falls eine Terminverschiebung notwendig wird. Die Beifügung einer Aufklärungsschrift zur ärztlichen Schweigepflicht und dem Datenschutz speziell im Rahmen der Begutachtung hilft ebenfalls, das Vertrauen des Probanden zu gewinnen. All dies lässt sich mit einer Einbestellung in barschem Ton nicht erreichen, welche verständliche Ängste vor der Untersuchung bewirkt.

\section{Erstkontakt in der Praxis}

Auch der Kommunikation zwischen dem nichtärztlichen Assistenzpersonal und dem Probanden kommt ein wesentlicher Stellenwert zu. Eine freundliche Begrüßung verknüpft mit Informationen über das weitere Prozedere z. B. über die voraussichtliche Wartedauer und geduldige Beantwortung der fast immer gestellten Fragen sollten eine Selbstverständlichkeit sein.

\section{Erstkontakt mit dem Arzt, Aufklärung und Abklärung der Aktenlage}

Der ärztliche Sachverständige sollte bedenken, dass der erste Eindruck bei der persönlichen Begegnung mit seinem Probanden entscheidend sein kann. Der Arzt sollte diesen hereinholen, ihm nach Aufruf entgegen gehen oder ihn gar persönlich im Wartezimmer abholen und nach einer freundlichen - aber keineswegs überschwänglichen - Begrüßung in sein Sprechzimmer hineinbitten. Ratsam ist eine zwanglose und zugewandte Gesprächseröffnung z. B. mit der banalen Frage, wie denn die Anreise war, ob man den Weg gleich gefunden habe und sich von den Strapazen der Anreise schon ein wenig erholt habe. Eine solche Gesprächseröffnung vermeidet den Eindruck, man habe nur begrenzt Zeit.

Der Begrüßung folgt die bereits angesprochene Aufklärung des Probanden über Sinn und Zweck des Gutachtens, seine Rechte und Mitwirkungspflichten, um sodann zum Thema der Begutachtung überzuleiten. Ist es dem ärztlichen Sachverständigen gelungen, in dieser Eingangsphase kommunikativ das Eis zu brechen und das Vertrauen des Probanden zu gewinnen, sollte ein zwangloser Übergang zum eigentlichen Gutachtenthema möglich sein. Dabei bewährte es sich, dem Probanden in knappen Worten vorzutragen, was man den übersandten Aktenunterlagen entnehmen konnte, verknüpft mit der Bitte, zu prüfen, ob diese Informationen auch der Erinnerung des Probanden entsprechen. Dieser kann dann notwendige Korrekturen und Ergänzungen vornehmen, was gelegentlich zu einer erheblich verbreiterten Informationsbasis beim Sachverständigen führt.

Diese Strategie einer Gesprächseröffnung über den Akteninhalt muss bei dem Probanden den Eindruck vermitteln, dass der Arzt seinen Fall bereits wahrgenommen und verinnerlicht hat und ernsthaft einen sorgfältig abwägenden Prüfungsprozess anstrebt, in dem er auch den Probanden an der Verifizierung des Sacherhalts beteiligt. Dazu ist es unabdingbar notwendig, dass der ärztliche Sachverständige bereits vor diesem Kontakt mit seinem Probanden die Akte nicht nur gelesen, sondern einen zumindest stich-
Trauma Berufskrankh 2014 - 16[Suppl 1]:26-30

DOI 10.1007/s10039-013-1959-0

๑) Springer-Verlag Berlin Heidelberg 2013

\section{F. Schröter \\ Kommunikation zwischen Arzt und Patient. Aspekte bei der Begutachtung}

\section{Zusammenfassung}

Hintergrund. Die Kommunikation zwischen Arzt und Patient stellt eine unverzichtbare Säule für die gutachtliche Beurteilung dar. Ohne Kommunikation - nicht zuletzt auch mit dem Auftraggeber des Gutachtens - wäre eine gutachtliche Sachverhaltsüberprüfung nicht möglich.

Vorgehen. Basis der Kommunikation ist eine empathische Öffnung des Arztes gegenüber dem Probanden, um dessen Vertrauen zu gewinnen und hierauf aufbauend erinnerliche Sachverhalte seitens des Probanden zu explorieren, die für die gutachtliche Beurteilung einen wesentlichen Baustein darstellen. Dargestellt werden das strategische Vorgehen im Umgang mit dem gutachtlichen Probanden, aber auch die weitere Kommunikation mit der auftraggebenden Institution, die das Gutachten inhaltlich verstehen können muss, um daraus eine rechtliche Entscheidung ableiten zu können.

\section{Schlüsselwörter}

Gutachten - Kommunikation - Empathie . Vertrauensbildung $\cdot$ Strategie

\section{Communication between physician and patient. Aspects of the assessment}

\section{Abstract}

Background. The communication between physician and patient represents an indispensible component of expert medical assessment. Without communication, not least with the commissioning authority, an assessment of the facts in the case is not possible.

Approach. The basis of communication is an empathic introduction by the physician towards the patient in order to win trust and building on this to explore facts which can be remembered by the patient and which represent an essential foundation for the expert assessment. This article presents the strategic approach to dealing with the expert medical assessment of patients as well as the additional communication with the commissioning authority which must be able to understand the contents of the report in order to be able to derive a legal decision.

\section{Keywords}

Expert testimony - Communication . Empathy · Trust · Strategy 
wortartartigen Aktenauszug gefertigt hat, der ihm auch bereits geschrieben vorliegen sollte. Nur dann kann er auf das - u. U. gegenteilige - Vorbringen seines Probanden eingehen, das von ihm ausgewertete Schriftstück anhand der Blattzahlangabe sofort wiederfinden und inhaltlich mit dem Probanden besprechen. Auf diesem Wege lassen sich Widersprüchlichkeiten im aktenkundigen Sachverhalt fast immer problemlos aufklären. Erkennbar werden dabei auch ärztliche Sünden im kurativen Bereich bezüglich ungeschickter oder unzutreffender Formulierungen. Verbliebene Unklarheiten in der Akte lassen sich durch zielgerichtete Befragung und den nachfolgenden Rapport des Probanden fast immer ausräumen. Kurzum: Der Proband fühlt sich ernst genommen als Mitwirkender am Gutachtenprozess und nicht nur als Beteiligter, über den gewissermaßen gerichtet wird.

Fast immer ist es möglich, den Probanden zu einem angesprochenen Problemkreis reden und berichten zu lassen, da sich in der Regel die Mitteilsamkeit rasch erschöpft. In den eher seltenen Fällen einer unerschöpflichen Logorrhö bedarf es einer einfühlsamen Gesprächsführung mit möglichst präzisen Zwischenfragen, die sich im Idealfall mit ja oder nein beantworten lassen. Bei einem aggressiven Vorbringen des Probanden bewährte sich das in Watte laufen lassen, nämlich die freundliche Attitüde gleich so, als hätte man die Aggressivität gar nicht wahrgenommen. Dem Autor gelang es auf diesem Wege so gut wie ausnahmslos, die Aggressivität ins Leere laufen zu lassen, bis sie von allein versiegte. Die Contenance zu wahren zahlt sich aus!

Schon eher schwierig ist der Umgang mit sehr wortkargen - meist intellektuell knapp ausgestatteten - Probanden, die zudem häufig Mühe haben, die ärztlichen Fragen inhaltlich zu verstehen. Ein geduldiges Nachfragen führt in aller Regel zum Ziel, auch wenn z. B. die Frage nach dem gegenwärtigen Befinden einfach mit „beschissen" beantwortet wird.

Die Kommunikation mit dem Probanden erfolgt - und dessen sollte man sich stets bewusst sein - auch für den Auftraggeber, der die Informationen wahrnehmen, verstehen und werten muss. Insofern bewährte es sich, wesentliche Passa- gen des probandenseitigen Vorbringens, insbesondere seine Ausführungen zu den aktuellen Beschwerden in wörtlicher Rede in Gegenwart des Probanden abzudiktieren, verknüpft mit der Bitte, dass er bei einer fehlerhaften Wortwahl korrigierend eingreift. Auch das entspricht einer vertrauensbildenden Maßnahme, vermittelt aber gleichzeitig auch einen unverfälschten Eindruck des patientenseitigen Vorbringens beim Empfänger des Gutachtens. Die Neigung zu einer verkürzten, sogar schon als Diagnose formulierten Wiedergabe der patientenseitigen Beschwerden (z. B.: „... beklagt eine Lumboischialgie ...") entspricht einer groben Entstellung dessen, was der Proband wirklich sagte und damit zum Ausdruck brachte, $z$. B.: „... so ein Sausen vom rechten Ohr, den ganzen Rücken runter, da ist's da unten so schlimm und dann auch so in der Leiste, manchmal bis in die Wade ..... Ein solches polytopes Beschwerdebild, welches weder anatomisch noch pathophysiologisch eine Erklärung finden kann, weist aus sich heraus auf die psychosomatisch geprägte Genese hin, was ein erfahrener Richter, auch ein erfahrener Sachbearbeiter bei der Versicherung, ebenfalls zu erkennen vermögen.

\section{Klinische Untersuchung}

Im Rahmen der klinisch-funktionellen Untersuchung sollte der Proband höflich zu den erwünschten aktiven Funktionsprüfungen gebeten werden. Sofern dabei Nachhilfe notwendig ist, sollte dies humorvoll geschehen (,lange nicht mehr geübt“?). Bei passiven Gegenprüfungen empfiehlt sich die Verwicklung des Probanden in ein Gespräch, was ablenkt und einem bewusstseinsnahen Gegenarbeiten entgegensteuert. Auf diesem Wege gelingt es fast ausnahmslos, die notwendigen klinischen Untersuchungen und Funktionsprüfungen soweit durchzuführen, dass man über belastbare und damit auch verwertbare Befunde verfügt.

\section{Abschluss}

Das Abdiktat der Befunde sollte erst nach Beendigung des Kontakts mit dem Probanden erfolgen, sinnvoll strukturiert sein, und man sollte sich einer absolut sachlichen Wortwahl befleißigen. Wurden simulative Momente erkannt, lässt sich dies auf dem Wege einer vollkommen nüchternen Beschreibung nicht vereinbarer Befundbeobachtungen, ggf. verknüpft mit dem Hinweis auf eine „bewusstseinsnahe Befundakzentuierung “ wiedergeben. Der stigmatisierende Begriff einer Simulation kann auf diesem Wege problemlos vermieden werden.

\section{Kommunikation mit der auftraggebenden Institution}

Die auftraggebende Institution (Gericht, Versicherung usw.) kann ein Gutachten nur verwenden, also in eine Entscheidung umsetzen, wenn der Empfänger dieses auch inhaltlich durchdringen und verstehen kann.

Die Vermittlung von gutachtlichen Aussagen an den nichtärztlichen Empfänger des Gutachtens kann nur unter Nutzung der deutschen Sprache, soweit dies irgend möglich ist, gelingen. Wird diese Anforderung an die Verständlichkeit eines Gutachtens missachtet und werden lateinische Fachausdrücke, insbesondere bei Befunden und Diagnosen, hemmungslos genutzt, ohne sie auch nur ansatzweise - was beim ersten Gebrauch des Begriffs ausreichend wäre - zu erläutern, zwingt dies den Empfänger des Gutachtens, in eine Übersetzung einzutreten, gestützt auf den Pschyrembel [3], was - wie gelegentlich in Gerichtsakten nachzuvollziehen - zu grotesken Entstellungen dessen führt, was der ärztliche Sachverständige zum Ausdruck bringen wollte. Dem Verfasser sind fehlerhafte Gerichtsentscheidungen bekannt, die auf eine solche Unzulänglichkeit des medizinischen Sachverständigen zurückzuführen sind. Werden sie, zumindest von einer der beiden Parteien, erkannt, besteht die Chance einer Revision vor einem Obergericht. Ansonsten ist der Sachverständige - und nicht das Gericht - Verursacher einer Fehlentscheidung, was dem Rechtsfrieden nicht dienlich sein kann.

\section{Nachgutachtliche Kommunikation}

Wurde der zu untersuchende Proband zu Beginn der Untersuchung korrekt über alle Besonderheiten des Gutachtens aufgeklärt, sollte er wissen, dass er seine Zwei- 
fel und eventuelle Rügen zum Gutachten nicht gegenüber dem Gutachter, sondern gegenüber dem Auftraggeber vortragen muss.

Wird derartiges vom Probanden direkt an den ärztlichen Sachverständigen herangetragen, wird er in dieser Situation die Kommunikation verweigern müssen, mit dem Hinweis gegenüber dem Probanden, dass man die Beschwerde an die auftraggebende Institution weitergeleitet hat. Sofern diese Institution hieraus Rückfragen an den Sachverständigen ableitet, sind sie jedoch selbstverständlich umfassend und - in deutscher Sprache - auch für den Probanden verständlich zu beantworten. Dabei sollte man sich tunlichst einer Wertung eines noch so unsinnigen Vorbringens enthalten, also nur in gebotener Sachlichkeit entgegnen.

Die auftraggebende Institution hat ein Recht auf Klärung der Sachverhalte, der Proband ein Recht darauf, es zu verstehen - aber eben nur auf diesem Wege.

\section{Resümee}

Eine gekonnte Kommunikation im Rahmen der medizinischen Begutachtung ist der Schlüssel für die Offenlegung aller in die Beurteilung einfließenden Einzelaspekte und unverzichtbar. Ein ärztlicher Sachverständiger, der diesen Anforderungen nicht gerecht werden kann, sollte sich anderen Aufgaben zuwenden, um der Gefahr von Fehlbeurteilungen infolge informativer Defizite aus dem Weg zu gehen.

Kommunikation kann man üben, dies - ganz banal - im normalen Lebensalltag, zumindest dann, wenn man sich bemüht, mit verständigen Menschen auf Augenhöhe zu kommunizieren. Von dieser Einstellung gegenüber einem Mitmenschen muss man überzeugt sein, um sie glaubhaft vertreten zu können. Diese Art des Umgangs miteinander könnte das Bild des idealen Arztes - und nicht nur des Gutachters - prägen im Sinne einer empathischen Zuwendung durch kommunikatives Geschick. So schrieb der Nobelpreisträger Bernard Lown in seinem Buch „Die verlorene Kunst des Heilens" [2], dass die Kommunikation auch die Qualität ärztlichen Handels erheblich erhöht, z. B. mehr als 75\% der Diagnosen durch Zuhören im Rahmen einer gekonnten anamnestischen Exploration gestellt werden können, weit mehr, als mit moderner Medizintechnik erreichbar. Dass dies auch dem überwiegenden Patientenwillen entspricht, lässt sich in einer vor wenigen Jahren durchgeführten europäischen Studie des Instituts für angewandte Qualitätsforderung und Forschung im Gesundheitswesen [4] nachlesen - kostengünstige und solide Medizin zugleich!

\section{Korrespondenzadresse}

\section{Dr. F. Schröter}

Interdisziplinäre Begutachtungen, IMB Kassel, Landgraf-Karl-Straße 21, 34131 Kassel

f.schroeter@imb-kassel.de

Interessenkonflikt. Der korrespondierende Autor gibt an, dass kein Interessenkonflikt besteht.

The supplement containing this article is not sponsored by industry.

\section{Literatur}

1. Bahrs O (2003) Mein Hausarzt hat Zeit für mich Wunsch und Wirklichkeit; Ergebnisse einer europäischen Gemeinschaftsstudie. GGW 3,1:17-23

2. Lown B (2007) Die verlorene Kunst des Heilens - Anleitung zum Umdenken, 2. Aufl. Schattauer, Stuttgart New York

3. NN (2012) Pschyrembel - klinisches Wörterbuch. deGruyter, Berlin

4. Van den Brink-Muinen A, Verhaak PFM, Bensing JM et al (1999) The Eurocommunication Study. An international comparative study in six European countries on doctor-patient communication. $\mathrm{NI}$ VEL, Utrecht 